\title{
The expression and significance of CD28, Ctla-4, CD80 and CD86 in ankylosing spondylitis were also stimulated.
}

\author{
Wanglei Du${ }^{1 \#, ~ L i n x u a n ~ P a n g ~}{ }^{1 \#, ~ Y a n n a ~ B a ~}{ }^{1}$, Tingting Lv $^{2}$, Zhaohui Zheng ${ }^{1}$, Xueyi Li ${ }^{1}$, JIN Ding ${ }^{1}$, \\ Guangzhi Xiao" ${ }^{1}$, Ying Li ${ }^{1}$, Ronghua Xie ${ }^{1}$, Ping Zhu ${ }^{1}$, Xichao Yang ${ }^{*}$, Zhenbiao Wu ${ }^{\text {* }}$ \\ ${ }^{1}$ Department of Clinical Immunology, Xijing Hospital, the Fourth Military Medical University, Shaanxi, Xi'an, PR \\ China \\ ${ }^{2}$ Department of Clinical Immunology, Tangdu Hospital, the Fourth Military Medical University, Shaanxi, Xi'an, PR \\ China
}

\#These authors contributed equally to this work

\begin{abstract}
Objective: To explore the expression and significance of CD28, ctla-4, CD80 and CD86 in ankylosing spondylitis.

Methods: Flow cytometry was used to test in January 2016-January 2017 in our hospital was 73 cases of ankylosing spondylitis patients and 40 normal controls CD28, CTLA 4, CD80 and CD86 weeks outside expression in lymphocytes. By ELISA method, the $\operatorname{lgA}$ serum immunoglobulin IgG, IgM and hypersensitive c-reactive protein (hs CRP) and blood sedimentation (ESR) levels, and to explore, CD80, CD86 and CD28, CTLA - 4, age, duration of the hs CRP, ESR, Bath AS functional index (BASFI) and Bath AS measurement index (BASMI) relevance.

Results: The levels of CD28, ctla-4, CD80 and CD86 were significantly higher in patients with ankylosing spondylitis $(\mathbf{P}<\mathbf{0 . 0 5})$. The levels of IgG and IgA in patients with ankylosing spondylitis were higher than those in the control group, and the difference was statistically significant $(P<0.05)$. The CD28 level of peripheral blood was positively correlated with ESR and BASFI index $(P<0.05)$, which was negatively correlated with hs-crp and BASMI index $(\mathbf{P}<0.05)$. The ctla-4 has negative correlation with ESR, hs-crp and BASMI index $(\mathbf{P}<0.05)$, and has no relationship with the BASFI index. CD80 was negatively correlated with ESR, BASFI index and BASMI index $(P<0.05)$, and had no relationship with hs-crp. CD80 has positive correlation with hs-crp and BASMI index $(\mathrm{P}<0.05)$, which has no relation with ESR and BASFI index.

Conclusion: The patients with ankylosing spondylitis is a total stimulus molecular CD28, CTLA 4, higher CD80, CD86 clear expression, the body is in a state of immune activation, has close relationship with immune dysfunction, monitoring peripheral CD28, CTLA 4, CD80, CD86 level is helpful for the early detection of disease, determine the illness treatment strategy.
\end{abstract}

Keywords: Co-stimulus, CD28, CTLA 4, CD80, CD86, Ankylosing spondylitis.

Accepted on November 13, 2017

\section{Introduction}

Ankylosing spondylitis is a kind of sub-type chronic progressive spinal joint diseases, it mainly invades spinal cord, relating to sacroiliac joint, joint axis and peripheral joint, is a kind of autoimmune diseases with the main symptom of constant inflammation [1]. Inflammation in high activity period and low activity appear alternately.

The incidence of this disease is increasing gradually. Omission missed diagnostic rate and error diagnostic rate are high. The treatment difficulty is high in the later stage. This disease still has a certain disability rate. The specific pathogenesis and onset mechanism are still unclear. At present, incidence of this disease has much close relation with heritage, chronic infection, autoimmune function disorder and endocrine disorder and so on, usually in male young adults. The ratio between male and female are 2:1.

Ankylosing spondylitis will cause disorder of work and life of patients. The study finds that ankylosing spondylitis patients will have abnormal cellular immune and liquid immune. Bone, joint and synovial tissue has lots of inflammatory $\mathrm{T}$ cells, mononuclear macrophage infiltration, $\mathrm{T}$ cell response and Th1/Th2 cell factors balance and metastasis [2]. Costimulus system has relations with immune function, lymph cells, 
especially T lymph cells directly. Costimulus molecular CD28, CTLA-4, CD80 and CD86 play important role in onset mechanism of ankylosing spondylitis [3]. Therefore, we use FCM to detect peripheral blood lymph cells phenotype and costimulatory molecular CD28, CTLA-4, CD80 and CD86 of 73 ankylosing spondylitis patients and 40 healthy people, the purpose is to learn the expression and meaning of costimulatory molecule in ankylosing spondylitis, providing new thinking for revealing onset mechanism of ankylosing spondylitis.

\section{Materials and Methods}

\section{Study objects}

Ethical approval was given by the medical ethics committee of Yan'an People's Hospital with the following reference number: 2015009,73 ankylosing spondylitis patients with the first visit from January, 2016 to January, 2017 in our hospital were selected as the study objects, all met the New York criteria issued in 1984 [4]. There were 54 male cases and 19 female cases. The age was from 16 to 54 years old. The average age was $26 \pm 8.2$ y old. This study selected 40 healthy people without rheumatic and spinal cord arthritis as the control group. There were 36 male cases and 14 female cases. The age was from 17 to $54 \mathrm{y}$ old. The average age was $25 \pm 8.9 \mathrm{y}$ old.

\section{Exclusive criteria}

(1) This study not used hormone and immune regulatory agent before sample collection; (2) Patients who had dysfunction of liver function and kidney function caused by liver and kidney diseases; (3) malignant tumor patients.

\section{Methods}

Collection and management of sample: All subjects were given $2 \mathrm{ml}$ fasting blood, and then it was input into heparin anticoagulation tube, located at home temperature for storing. Then this study used FCM to do analysis and management within $24 \mathrm{~h}$. In addition, this study adopted $2 \mathrm{ml}$ whole blood to separate serum, and then it was located at $4^{\circ} \mathrm{C}$ for storing for serum immune globulin, hs-CRP and ESR measurement and so on. Recording BASFI and BASMI of all patients.

FCM analysis: This study selected $100 \mu \mathrm{l}$ anticoagulation whole blood, they were marked by CD28, CTLA-4, CD80 and CD86 to do multiple color flurorescent, then this study mark corresponding Ig G-FITC/Ig G1, PE/Ig G1, Cy-chrome /Ig G1APC antibody as negative control, it mixed evenly and incubated for $20 \mathrm{~min}$ at home temperature away from light. Then this study used lxFACS hemolytic agent, it was given phosphate buffer $\mathrm{S}$ once after hemolysis and then computer test. Then this study used SFC-SSC to set distribution of lymph cells on scatter diagram, calculate percentage of CD28, CTLA-4, CD80 and CD86 cells accounting for lymph cells. Analyzing 5000 cells in each sample.

Serum immune globulin measurement: This study used ELISA to detect concentration of IgG, IgA, IgM, hs-CRP and ESR after serum separation.

\section{Statistical methods}

This study used SPSS 17.0 to do statistical analysis for study results. Measurement data were represented by $\bar{x}$ s. It used ttest. Relevant parameter of clinical character in ankylosing spondylitis and CD28, CTLA-4, CD80 and CD86 used Pearson analysis to test c. $\mathrm{P}<0.05$, there were statistical differences.

\section{Results}

\section{Globulin level in serum in two groups}

IgG and IgA level in serum of ankylosing spondylitis patients higher than the control group, there were statistical differences $(\mathrm{P}<0.05)$. IgM in ankylosing spondylitis patients higher than the control group, but there were no statistical differences $(\mathrm{P}>0.05$, Table 1).

Table 1. Comparison of globulin protein in serum in two groups $(\bar{x} \pm$ s).

\begin{tabular}{lllll}
\hline Group & Cases & $\lg \mathbf{g}(\mathbf{g} / \mathbf{L})$ & $\operatorname{IgA}(\mathbf{g} / \mathbf{L})$ & $\lg \mathbf{M}(\mathbf{g} / \mathbf{L})$ \\
\hline The observation group & 73 & $14.7 \pm 3.27^{*}$ & $3.31 \pm 1.72^{*}$ & $1.84 \pm 0.76$ \\
\hline The control group & 40 & $11.2 \pm 3.21$ & $2.45 \pm 1.25$ & $1.79 \pm 0.67$ \\
\hline Note: ${ }^{*} \mathrm{P}<0.05$ & & & & \\
\hline
\end{tabular}

\section{Comparison of peripheral blood costimulatory molecule in two groups}

CD28, CTLA -4, CD80 and CD86 level of ankylosing spondylitis patients higher than the control group obviously, there were statistical differences $(\mathrm{P}<0.05$, Table 2$)$.

Table 2. Comparison of peripheral costimulatory molecule in two groups $(\bar{x} \pm s)$.

\begin{tabular}{|c|c|c|c|c|c|}
\hline Group & Cases & CD28 & CTLA-4 & CD80 & CD86 \\
\hline The observation group & 73 & $12.87 \pm 0.73^{*}$ & $10.22 \pm 1.65^{*}$ & $10.08 \pm 4.69^{*}$ & $14.35 \pm 2.86^{*}$ \\
\hline The control group & 40 & $7.68 \pm 3.82$ & $4.78 \pm 1.24$ & $7.25 \pm 3.13$ & $9.86 \pm 1.36$ \\
\hline
\end{tabular}




\section{Comparison of CD80/CD86 and CD28/CDCTLA-4 in two groups}

CD80/CD86 and CD28/CTLA-4 of ankylosing spondylitis patients lower than the control group, but there were no statistical differences $(\mathrm{P}>0.05$, Table 3$)$.

Table 3. Comparison of CD80/CD86 and CD28/CDCTLA-4 in two groups $(\bar{x} \pm s)$.

\begin{tabular}{llll}
\hline Group & Cases & CD80/CD86 & CD28/CTLA-4 \\
\hline The observation group & 73 & $0.71 \pm 0.05$ & $1.07 \pm 0.07^{*}$ \\
\hline The control group & 40 & $0.74 \pm 0.12$ & $1.26 \pm 0.37$ \\
\hline Note: ${ }^{*} P<0.05$ & & & \\
\hline
\end{tabular}

\section{Relevant parameter of clinical character of ankylosing spondylitis patients and $C D 28, C T L A-4$, CD80 and CD86}

CD28 level in peripheral blood of ankylosing spondylitis patients had positive correlations with ESR and BASFI indexes $(\mathrm{P}<0.05)$, had negative correlations with Hs-CRP and BASMI indexes $(\mathrm{P}<0.05)$. CTLA-4 had negative correlations with ESR, Hs-CRP and BASMI indexes $(\mathrm{P}<0.05)$, no correlations with BASFI indexes. CD80 had negative correlations with ESR, BASFI and BASMI indexes had no correlations with ESR and BASFI indexes (Table 4).

Table 4. Relevant parameter of clinical characters of ankylosing spondylitis patients and CD28, CTLA-4, CD80 and CD86.

\begin{tabular}{lllll}
\hline Observation indexes & CD28 & CTLA-4 & CD80 & CD86 \\
\hline Disease history $(\mathrm{y})$ & -1.42 & 0.07 & -0.34 & -0.63 \\
\hline Age & -0.65 & 0.09 & -0.38 & -0.67 \\
\hline ESR $(\mathrm{mm} / \mathrm{h})$ & $0.648^{*}$ & $-0.427^{*}$ & $-0.325^{*}$ & -0.221 \\
\hline Hs-CRP $(\mathrm{mg} / \mathrm{L})$ & $-0.588^{*}$ & $-0.328^{*}$ & 0.179 & $0.127^{*}$ \\
\hline BASFI $(0 \sim 10)$ & $0.884^{*}$ & -0.431 & $-0.265^{*}$ & -0.326 \\
\hline BASMI $(0 \sim 10)$ & $-0.726^{*}$ & $-0.681^{*}$ & 0.257 & $0.217^{*}$ \\
\hline
\end{tabular}

Note: ${ }^{*} \mathrm{P}<0.05$

\section{Discussion}

Abnormal cellular, immune regulation and immune function severely influence the incidence and development of ankylosing spondylitis with injury of chronic spinal cord arthritis. Study on T cell activation is still the core problem of immune fields. The effects of costimulatory CD28, CTLA-4, CD80 and CD86 participating in $\mathrm{T}$ cell activation and abnormal function are still the study hotspot problems at present. The activation of $\mathrm{T}$ cell need two signal from cells outside [5]. On the one hand, it needs the specific antigen peptide compounds of initial Signal MHC molecule, though initial signal is necessary in the activation of $\mathrm{T}$ cells, but it can't promote proliferation of $\mathrm{T}$ cells and secretion of cellular factors; on the other hand, it needs costimulatory signals, mainly in T cells of membrane protein molecular surface and stimulatory signal induced by ligands. It is the necessary signal of complete activation $\mathrm{T}$ cells after recognizing antigen. Initial signal can stimulate primary activation of $\mathrm{T}$ cells, furtherly simulate primary activation of $\mathrm{T}$ cells into full activation, proliferation and differentiation stages, thus, playing immune effects [6].

At present, this study finds that costimulatory molecule include B7 (CD80/86)/cD28, CD54/LFA-1, CDZ/LFA-3 (CD58), CD40/CD154, CD24/CD24L and so on. The most important is CD28 in surface of $\mathrm{T}$ cells, CTLA-4 (CD152) and corresponding ligand CD80 and CD86 of APC surface. CD28 provides $\mathrm{T}$ cell activation signal. CD80 and CD86 provide feedback immune regulatory signal, thus playing inhibition.

$\mathrm{T}$ cells will lose immune function of there are only initial signal but no costimulatory signal, and it cannot enter into reaction state or change into immune tolerance state, even apoptosis. At the same time, over-strong costimulatory signal will activate $\mathrm{T}$ cells excessively, causing autoimmune diseases [7,8]. Costimulatory molecular CD 28 combines with CD80, it is the cellular immune response induced by assistant $\mathrm{T} 1$ cells, which promotes Th1 cell proliferation. CD86 has relations with humoral immune response induced by Th2, it combines with CD28, finally promoting Th1 cell proliferation [9]. CD-28 and CD-86 as $\mathrm{T}$ cell costimulatory molecule which are studied widely, it can express in various APC, such as dendritic cells, langerhans cells, activation macrophage cells, B cells and so on, they two combine with different receptors, stimulatory CD28 and inhibiting CTLA-4 [10]. The structure of CD 28 and CTLA-4 are similar, but there are pair of costimulatory molecules which have positive and inverse regulatory function. On one hand, it manifests on the difference of CD28 and CTLA-4 expression dynamics, CD28 is the constitutive expression on $\mathrm{T}$ cell surface, and after $\mathrm{T}$ cell activation, CTLA-4 can be upgraded rapidly. Comprehensive ability of CD-86 is 13 times weaker than CD28, effective combination of CD28 is twice to three times greater than CD-86 [11]. At the same time, CD28 is the inducing expression on APC, the time of expression up-regulation by induction is later than D-86. CD-86 is the low constitutive expression, it can be upregulated rapidly. CD28 combines with ligand to provide $\mathrm{T}$ cell signal activation. CD-86 can provide feedback immune regulatory signal, playing inhibition [12]. Study on CD-86 and $\mathrm{CD} 80$ are the hotspot of autoimmune diseases at home and abroad.

This study use fluorescence quantitative PCR to detect CD80, CD86, CD28 and CTLA-4, it is a new method. Studies find that CD80 and CD86 expression on surface of peripheral blood mononuclear cells of ankylosing spondylitis higher than the control group obviously. CD28 expression higher than the normal control group obviously, it shows that ankylosing spondylitis patients will have over-expression caused by CD80 and CD86 on surface of APC, which will provide adequate ligands to $\mathrm{T}$ cells. At the same time, corresponding receptor CD28 on surface of $\mathrm{T}$ cells also express excessively. 
Combination of those two can produce adequate costimulatory signal, activate $\mathrm{T}$ cells excessively. It will induce rheumatoid arthritis and allergic purpura etc. which relate to immune deficiency [13-15]. It can be guessed that incidence of ankylosing spondylitis has direct relations with immune state of body disorder. At the same time, in Table 1, we can see that Igc and $\lg$ A immune globulin protein of ankylosing spondylitis patients higher than the normal control group [9] obviously, the main reason is CD28 induced by activation T cells can promote $\mathrm{B}$ cell clone and transformation of expression CD 86 and antibody production [16-18]. CD80/CD86 ratio lower than the normal group, which is in accordance with balance and metastasis of Th1 and Th2 cellular factors. CTLA-4 expression of ankylosing spondylitis patients higher than the normal control group, but its CD28/CTLA-4 lower than the normal control group obviously. Therefore, we can judge that main ligands of CTLA-4 are CD80. Main ligand of CD28 is CD86. When CD86 expression is high on surface of APC, the effects of CD86-CD28 are more dominate comparing with CD80CTLA-4 to activate $\mathrm{T}$ cells. This mechanism plays an important role in the onset process of ankylosing spondylitis.

This study explores correlations between CD28, CTLA-4, CD80 and CD86 of ankylosing spondylitis patients and clinical character. CD28 level has positive correlations with ESR and BASFI indexes $(\mathrm{P}<0.05)$, has negative correlations with HsCRP and BASMI indexes $(\mathrm{P}<0.05)$. CTLA-4 has negative correlations with ESR, Hs-CRP and BASMI indexes $(\mathrm{P}<0.05)$; CD80 has negative correlations with ESR, BASFI and BASMI indexes $(\mathrm{P}<0.05)$; $\mathrm{CD} 80$ has positive correlations with Hs-CRP and BASMI indexes $(\mathrm{P}<0.05)$. CD28, CTLA-4, CD80 and CD86 level have no correlations with age and disease history. Clinical studies find that rise of CD28, CTLA-4 and CD80 can reflect the severity of disease history.

In conclusion, this study explores the onset mechanism of ankylosing spondylitis through detecting expression form of CD28, CTLA-4, CD80 and CD86 and from cellular immune perspective, which reveals incidence and development of ankylosing spondylitis have close relations with abnormal expression of costimulatory molecule CD28, CTLA-4, CD80, CD86. So immune regulation and intervention can provide new thinking and indexes for diagnosis, prognosis and treatment of ankylosing spondylitis.

\section{References}

1. Zhang P, Martin M, Yang QB. Role of B7 costimulatory molecules in immune responses and $\mathrm{T}$ - helper cell differentiation in response to recombinant $\mathrm{Hag} \mathrm{B}$ from Porphyromonas gingivalis. Infect Immun 2004; 72: 637-644.

2. Wang B, Wang PP. Study progress of onset mechanism of ankylosing spondylitis. Sichuan Med J 2008; 29: 1429-1430.

3. Wang LL, Cai B, Fen WH. Study on abnormal T cell regulation and immune function disorder of SLE and rheumatoid arthritis. Checkout Med 2006; 21: 95-99.
4. Vander Linden SJ, Valkenburg HA, Cats A. Evaluation of diagnostic criteria for ankylosing spondylitis: a proposal for modification of the New York criteria. Arthritis Rheumatis 1984; 27: 361-368.

5. You J B, Mao XH, Dong ZQ. Analysis of $\mathrm{T}$ cell sub immune inflammation indices in peripheral blood of HLAB27 + ankylosing spondylitis and non-ankylosing spondylitis patients. Chinese J Health Lab Technol 2016.

6. Suvas S, Singh V, Sahdev S. Distinct role of CD80 andCD86 in the regulation of the activation of B cell and B cell lymphoma. J Biol Chem 2002; 277: 7766-7775.

7. Bourgeois C, Rocha B, Tanchot C. A role for CD40 expression on $\mathrm{CD} 8+\mathrm{T}$ cells in the generation of $\mathrm{CD} 8+\mathrm{T}$ cells memory. Science 2002; 297: 2060-2063.

8. Ke XY, Gribben J, Wang J. The identical effects of B7-1 and B7-2 on regulation of human IL-2 gene transcriptionfactors NF-kappa B and AP-1. Zhongguo Shi Yan Xue YeXue Za Zhi 2002; 10: 512-518.

9. Willemsen RA, Ronteltap C, Chames P. T cell retargeting with MHC class I-restricted antibodies: the CD28costimulatory domain enhances antigen-specific cytotoxicity and cytokine production. J Immunol 2005; 174: 7853-7858.

10. Afsharsterle S, Zotos D, Bernard NJ. Fas ligand-mediated immune surveillance by $\mathrm{T}$ cells is essential for the control of spontaneous B cell lymphomas. Nat Med 2014; 20: 283-290.

11. Zhang N, He YW. The antiapoptotic protein Bcl-x L is dispensable for the development of effector and memory tlymphocytes. J Immunol 2005; 174: 6967-6973.

12. Salomon B, Bluestone JA. Complexities of CD28/B7: CTLA-4 costimulatory pathways in autoimmunity and transplantation. Annu Rev Immunol 2001; 19: 225-252.

13. Ma ZX, Zu Y, Bao YM. Expression of costimulatory molecules of peripheral blood mononuclear cells in children with henoch schonlein purpura and its significance. J Appl Clinic Pediatr 2008; 23: 1654-1657.

14. Cai B, Wang LL, Chen J. Imbalance of costimulatory molecules and $\mathrm{T}$ lymphocytes in patients with rheumatoid arthritis. Immunol J 2005; 21: 160.

15. Lu M, Ye JR, Zhang YG. Expression of CD28 and inducible co-stimulator on $\mathrm{T}$ lymphocytes in peripheral blood of patients with atopic dermatitis. Sichuan Med J 2009; 30: 726-728.

16. Leibson PJ. The regulation of lymphocyte activation by inhibitory receptors. Curr Opin Immunol 2004; 16: 328-336.

17. Iñiguez $M A$, Punzon $C$, Nieto $R$. Inhibitory effects of sigma-2 receptor agonists on $\mathrm{T}$ lymphocyte activation. Front Pharmacol 2013; 4: 23.

18. Schulz K, Frahm J, Kersten S. Effects of inhibiting dipeptidyl peptidase-4 (dpp4) in cows with subclinical ketosis. Plos One 2015; 10: 0136078.

\section{*Correspondence to}

Xichao Yang 
Department of Clinical Immunology

Xijing Hospital

The Fourth Military Medical University

PR China

Zhenbiao $\mathrm{Wu}$
Department of Clinical Immunology

Xijing Hospital

The Fourth Military Medical University

PR China 